\title{
SHAPE MEMORY POLYMERS: A JOINT CHEMICAL AND MATERIALS ENGINEERING HANDS-ON EXPERIENCE
}

\author{
Mujan N. Seif and Matthew J. Beck \\ University of Kentucky • Lexington, KY 40506
}

N tudent retention is an ongoing area of concern for engineering programs nationwide. ${ }^{[1-5]}$ Although it is generally accepted that both the difficulty of engineering coursework and changes in student career interests decrease engineering retention rates, these are not factors academic institutions can easily control. ${ }^{[-8]}$ However, engineering programs do have the opportunity to emphasize the real-world importance and impact of engineering practice immediately upon students' arrival in an effort to establish genuine enthusiasm early in students' college careers ${ }^{[7-10]}$ While challenging texts and difficult problem sets may dim the fervor of even the most prepared freshmen, providing opportunities to see how knowledge can directly yield practical outcomes can motivate students from all backgrounds. ${ }^{[1-13]}$ More specifically, it has been widely shown that hands-on experiences highlighting complex engineering concepts are "best practices" for engaging and informing beginning engineering students..$^{[4,9,10,14-21]}$

While igniting interest in new engineering students is important for improving student retention and outcomes, equally so is promoting cooperation among students. As rapid and rich communication becomes less costly, more reliable, and more readily available to stakeholders across the globe, the ability to collaborate across disciplines and backgrounds while solving engineering problems has become critical to the modern practice of engineering ${ }^{[18,22-25]}$ Engineers in different fields and at distant locations now have ample opportunity to combine their respective expertise and pursue ideas that are only feasible collaboratively. From this perspective, cross-disciplinary experiences should be available to students throughout their academic studies. ${ }^{[24-26]}$ Cross-disciplinary labs and demonstrations not only foster collaboration skills, but also allow students to make better-informed decisions regarding their own interests and goals.

Addressing this need for engaging and cross-disciplinary student experiences, we describe a joint chemical and materials engineering hands-on experience centered on the synthesis and physical characterization of a shape-memory polymer. Polymer science is an area of strong overlap between chemical and materials engineering, and is therefore a promising base from which to engage students in both disciplines and motivate cross-disciplinary discussion. In addition, "shapememory" polymers are an exciting new class of materials with a host of promising applications (see, e.g., Reference 27).

\section{Mujan N. Seif received her B.S. in materials engineering from the University of Kentucky in May 2017. She has been heavily involved in UK's Material Advantage chapter as well the Society of Women Engineers. She con- tinues to be active in SWE as a member of its Collegiate Leadership Institute. She previ- ously completed a co-op at DuPont Titanium Technologies, and is doing research in Dr. Katsuyo Thornton's Computational Materials group at the University of Michigan during the 2017-2018 academic year.}

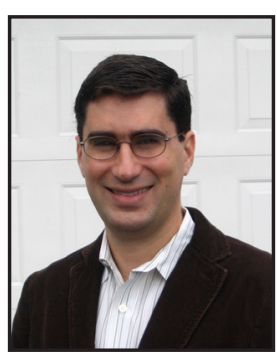

Matthew J. Beck is an associate professor in the Chemical and Materials Engineering Department at the University of Kentucky. In 2015 he won the Provost's Outstanding Teaching Award. He is heavily involved in recruiting and outreach activities on behalf of the Materials Engineering program, and currently serves as the co-director of Graduate Studies in the Chemical and Materials Engineering Department. He received his $P$ h. D. in materials engineering from Northwestern University, and his research group uses atomistic calculations to probe the structure and properties of nanomaterials and surfaces for applications in catalysis, energy generation and storage, and electron beam generation.

(๑) Copyright ChE Division of ASEE 2018 
In the following we describe both a "demo" and an extended version of the experience appropriate for different groups of students. During a guided, hands-on "demo" experience, first-year engineering students combined liquid polymer precursors and a photo catalyst, and, using UV light to drive polymerization, synthesized a solid polymer sheet. Students then explored effects of a glass transition on mechanical properties of the polymer they synthesized.

As part of a sophomore lab course, students extended beyond the guided "demo" experience by exploring the effects of varying the initial mixture composition and processing time on final-state mechanical properties. Responsive write-ups prepared by participating students along with survey results were used to assess students' engagement and understanding of the connections between processing, structure, and properties of the polymer sheets.

\section{CONTEXT AND OBJECTIVES}

The Chemical and Materials Engineering Department at the University of Kentucky is home to separate undergraduate and graduate programs in chemical engineering (CME) and materials science and engineering (MSE). Over the past few years the CME and MSE programs have annually enrolled $\sim 120$ and $\sim 24$ new first-year students, respectively. During this time, each program has offered a required 1-credit introductory MSE 101 or CME 101 course to first-year students in their incoming fall semester. These courses are intended to introduce beginning students to the kinds of knowledge and work typical of their chosen discipline, and to motivate student interest in further coursework.

Beyond the MSE or CME 101 course, students in both programs primarily take core math, physics, and chemistry courses during their first academic year. In the fall of their second year, MSE students take a first materials engineering lab course (MSE 202) in parallel with Materials Science I (MSE 201) - a survey course based on Callister and Rethwisch's Fundamentals of Materials Science and Engineering: An Integrated Approach. The MSE 202 lab course focuses on introducing students to the techniques and equipment commonly used in materials engineering practice, as well as proper documentation in the form of lab notebooks and lab reports. Labs in MSE 202 are open-ended investigations focused on exploring how processing changes the structure and properties of different materials. Students are expected to apply knowledge gained in the MSE 201 lecture course to analyze and evaluate observations made in the MSE 202 lab, and are assessed primarily on the basis of individual lab notebooks used during lab, and individual lab reports submitted one week after the lab. MSE 101 and 202 have been taught by the co-author (Beck) since Fall 2009 and 2010, respectively.

The activities described here were originally designed in Fall 2012 as a "hands-on experience" for MSE 101, intended to spark student interest through a real-world demonstration of how processing (in this case, exposure to UV light and, separately, changes in temperature) alters the structure and properties of polymeric materials. Beginning in Fall 2013, the experience was opened to first-year students in both MSE and CME 101 - a total of 140 students per year. Both CME and MSE 101 students were provided a 50-minute lecture introduction to the structure and properties of polymer materials prior to participation in the experience. This lecture defined polymers as $\mathrm{C}$-based chains of repeated monomers and discussed the role of entanglement and crosslinking with regard to the observable macroscopic stiffness of polymer materials. The lecture concluded with a guided discussion of the role temperature plays in influencing chain flexibility and, subsequently, mechanical stiffness.

In Fall 2014 an expanded version of the "hands-on experience" was included as a full lab investigation in the sophomore MSE 202 lab (enrollment: 20-25). Distinct from the demo version of the experience in MSE 101, student lab groups in MSE 202 were asked to measure properties of the polymers they synthesized as a function of the polymer composition, and to deduce empirical rules for the glass transition temperature and/or the degree of polymer crosslinking.

Beyond simply engaging students, both the demo (MSE 101) and full lab (MSE 202) versions of the experience were designed to reinforce polymers-related learning outcomes. Neither experience was intended to serve as a stand-alone module for holistic training in polymers processing and behavior. Even so, both experiences aimed to improve student understanding of:

- Safety procedures for volatile organics.

- Basic mixing and measuring procedures.

- The structure of polymer chains, polymer films, functional groups, and cross-links.

- The difference between polymerization and cross-linking.

- The general mechanism of photo-catalyzed reactions.

- Glass transitions in polymers, including the role of polymer structure/crosslinking in controlling viscosity and film mechanical properties.

During Fall 2015 the co-author (Seif), then a junior in the UK MSE program, completed an independent study project focused on assessing and optimizing the polymer synthesis process used during the experience. Her initial focus was assessing an alternative microwave heating process for activating cross-linking, which then transitioned to assessing a UV-initiated polymerization process that made use of commercially available acrylic nail lamps. The results and findings of both investigations are presented in the Appendix.

\section{PROCEDURES}

\section{Polymer Synthesis}

To form a solid co-polymer film with a glass transition temperature of $\sim 50{ }^{\circ} \mathrm{C}$, liquid poly (methyl methacrylate) (MW 100, purchased from Acros Organics) and poly(ethylene 
glycol) (MW 750, purchased from Aldrich Chemistry), PMMA and PEG, respectively, were combined with 2,2-Dimethoxy-2-phenylacetophenone (DMPA) photo-initiator. The base recipe used in the MSE 101 demo version was $15 \mathrm{~g}$ PMMA, $5 \mathrm{~g}$ PEG, and 0.1 g DMPA. During the MSE 202 full lab version, lab groups designed a set of approximately nine variations of the base recipe to explore the effects of variable composition on final polymer properties. DMPA was used in powder form, and the polymer solution was mixed in $50 \mathrm{~mL}$ Erlenmeyer flasks on a magnetic stir plate for 3 minutes to ensure complete DMPA dissolution and even mixing. Mixed batches of polymer precursor (approximately $30 \mathrm{~mL}$ ) were stored in stoppered flasks to prevent evaporation.

Molds for casting polymer sheets were constructed from either two $7.5 \mathrm{~cm}$ x $5 \mathrm{~cm}$ Pyrex microscope slides with nominal thicknesses of $1.0 \mathrm{~mm}$ (hands-on demo) or 4 in. $\mathrm{x} 6$ in. picture frame glass panels with nominal thicknesses of $1 / 16$ th inches (expanded laboratory). The slides were prepared for casting by washing in soap and water, then treated with commercially available RainX $\mathrm{X}^{\mathrm{TN}}$ per the manufacturer's instructions. The RainX coating was necessary to prevent adhesion between the UV-cured polymer film and the glass slides. U-shaped spacers were cut from Teflon sheets with nominal thicknesses of $0.8 \mathrm{~mm}$, purchased via McMaster-Carr. A stack of glass slide, teflon spacer, glass slide was then constructed and held together with regular binder clips (see Figure 1). In order to reduce spillage when the molds were laid face-down during UV curing; larger binder clips were used at the top (open end) of the stack.

Plastic pipettes were used to load precursor solution into the assembled molds. Molds were filled such that 1-2 cm of empty mold remained at the top. Loaded molds were immediately transferred into a commercially available USpicy 36W Nail Dryer/UV Lamp system (see Figure 2, and Appendix for more details). The lamps were turned on, and the polymer precursor cured under UVA (365 nm) exposure for 10 minutes. Once the UV light was turned off, the binder clips were removed and the glass slides separated by hand to release the solid polymer film. Glass slides and teflon spacers were washed in soap and water following each use. Slides were re-coated with RainX after approximately four uses.

\section{MSE 101 "Hands-On Demo" - Outline of Activities}

During each of the 2013-2015 Fall semesters, approximately 140 freshmen MSE and CME students participated in the hands-on demo. Each year the demo was offered over two 3.5 hour windows, with four students arriving to begin the experience as a group every 12 minutes. An online signup was used to allow students to select the time slot of their preference, and each student was instructed to expect to spend approximately 50 minutes completing the demo. To facilitate the experience, four to five MSE upper-division undergraduate students in conjunction with the course instructor (Beck) manned four work stations and a check-in station.

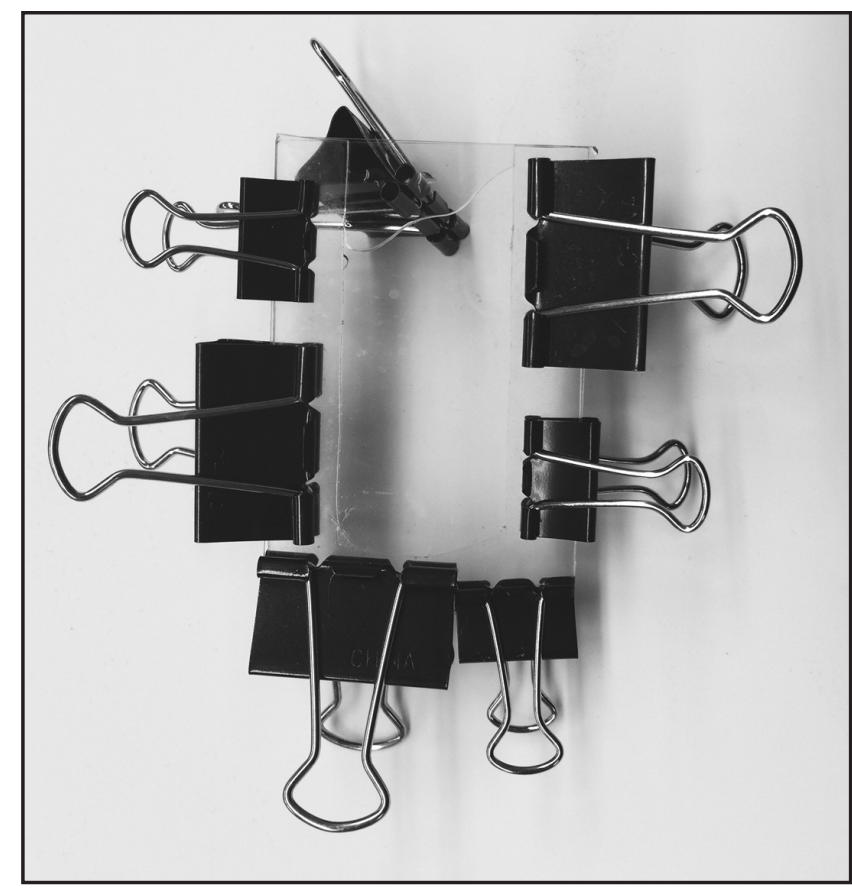

Figure 1. The structure of the mold assembly. Note that six binder clips are attached and the assembly is resting on the seventh to keep the contents from spilling.

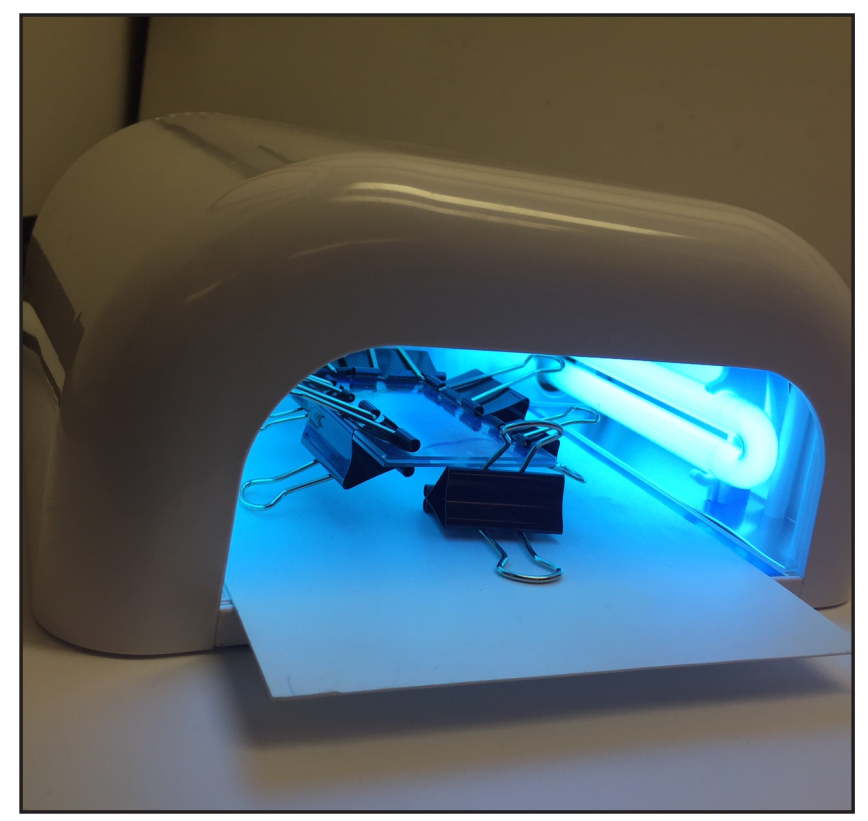

Figure 2. The assembly inside the USpicy acrylic nail lamp. Note the assembly is resting on a thick sheet of paper to ease transfer between stations.

At the check-in station, each participating student was provided a single-page outline of the demo including brief written instructions for each workstation, personal protection equipment, and a flask for their polymer blend (see Equipment Details, below). Arriving students did not necessarily know 
their groupmates, and before beginning their work a facilitator led the students through a brief ( $<5$ minutes) welcome and introduction. Students were advised to take notes on the back of their demo handout to prepare for writing a required one-page report describing their actions and discussing the processing-property relationships explored during the demo.

Each group of students then proceeded to the first workstation, "Mixing." Students used a balance to add the appropriate amounts of PEG-DMA, MMA, and DMPA to their flasks, and mixed the reagents with a magnetic bead on a magnetic stir plate. A facilitator discussed the definition of polymers and the different properties of the molecular precursors with the participating students while at the station. In particular, students were asked to consider the differing viscosities of (relatively high molecular weight) PEG-DMA and (low molecular weight) MMA, to highlight the relationship between viscosity and polymer chain length. Each group spent $\sim 10$ minutes at the Mixing station.

Students then moved to the "Mold Assembly" station, where they assembled their film mold from two Pyrex slides (previously coated with RainX), a U-shaped teflon spacer, and 6-8 small binder clips (see Figure 1). Larger binder clips were used near the top of the mold to prop up the open mouth of the mold and prevent mixture leakage during UV irradiation. After loading their mold using a plastic pipette, the mouth of the mold was covered with parafilm for transport to the next station. Groups spent 10 minutes at the Mold Assembly station. The third workstation was the "UV Irradiation" station. Students placed their filled mold into the light source and irradiated for 10 minutes. During irradiation, a facilitator discussed the role and mechanism of action of the photo-inititator (DMPA). Discussion focused on DMPA's capture of energy by absorption of UV photons, and the resulting breaking of bonds that then drive the formation of new cross-link bonds between polymer chains. A final note of emphasis addressed the effects of too few, or extra, cross-links on the stiffness and brittleness of the resulting solid polymer films.

At the final workstation, students removed their polymer film and washed it in soap and water to remove uncured polymer remaining on the surface. Then, using a heat gun and forceps, heated their film above the glass transition temperature $\left(\sim 50{ }^{\circ} \mathrm{C}\right)$, and observed the change in film stiffness. Students were able to bend their films and quench them in beakers of cold water, demonstrating that the films could be made to hold a new shape. Re-heating the films allowed them to unfold to their original flat film shape, and demonstrated the reversibility and repeatability of the glass transition in terms of mechanical stiffness.

To provide a comparison with another polymer, students were also given $8 \mathrm{~cm}$ by $10 \mathrm{~cm}$ pieces of commercially obtained polystyrene sheets, which, under similar heating as applied to their own polymer, irreversibly shrunk and became rigid (due to crystallization). A facilitator discussed the role of thermal energy in enabling chain flexibility and its relation to the glass transition. Discussion of the polystyrene focused on the fact that when heated, polystyrene chains are free to align with each other, and that this polymer crystallization results in irreversible densification and increased stiffness.

\section{MSE 202 "Laboratory Investigation" - Outline of Activities}

During Fall 2014 and 2015 an expanded version of the hands-on demo experience was offered as a full laboratory investigation in the sophomore MSE 202 lab course. Lab groups of three or four students were offered the same introduction handout provided to the MSE 101 participants. MSE 202 lab groups were then instructed to investigate the effects of varied reagent amounts and/or processing time on the mechanical properties and glass transition temperature of the synthesized polymers. Each group mapped out a set of approximately nine different recipe variations to test, varying the concentrations of one or more reagents, and/or the UV irradiation time. The experimental parameters were not assigned, but rather decided by the students themselves in discussion with their lab group. Polymer synthesis and characterization were carried out over two 3-hour meetings, and data analysis was conducted by students as homework.

MSE 202 lab groups followed the same basic processing steps as MSE 101 demo participants: blending reagents, casting a film between two glass sides, and UV irradiating samples in the USpicy 36W Nail Dryers. Once samples were fabricated, the qualitative effects of recipe variations on film properties and appearance were recorded by students. To quantitatively characterize their samples' mechanical properties, students performed tensile testing on an Instron 4400 Universal Testing System. Four-inch standard tensile "dog bones" (gauge length $40 \mathrm{~mm}$ ) were punch cut with a steel die on a hand-operated press. Clamp grips were mounted on the Instron, and extension tests run at between 1 and $5 \mathrm{~mm} / \mathrm{min}$ were conducted by the students. Load-displacement curves were recorded and subsequently converted to engineering stress-strain curves. Qualitative observations of fracture surfaces, including viewing of polymer samples on a light table between polarizing lens, were made and recorded.

To quantitatively determine the glass transition temperature, a hot plate was used to heat water in a beaker until a polymer sample placed in the water was clearly above the glass transition temperature (that is, was no longer stiff or rigid). Using a stir rod to constantly agitate and flex the film, the water bath and film were allowed to cool naturally in air while the temperature was continuously monitored with a thermocouple. The approximate temperature at which the film stiffened was recorded, and compared among films.

\section{Assessment Methodologies}

MSE 101 student engagement was assessed via an ungraded and anonymous online survey, and student learning 
outcomes were assessed via a graded one-page brief report. The report required students to describe what they did during the experience and highlight what they learned about the processing and properties of polymers.

MSE 202 student outcomes were evaluated via a graded full lab report due one week after the completion of the lab. The MSE 202 lab reports were formal technical reports including a formal title and header, an abstract, and five sections of main body text: introduction; background; procedures; results and discussions; and conclusions. For the purposes of this study, a subset of student artifacts were re-assessed independent of grade earned for class, focusing solely on whether the reports clearly demonstrated that the hands-on polymers experience itself motivated student engagement and/or achieved the learning outcomes described above.

To assess student achievement of learning outcomes based on MSE 202 lab reports, the degree to which each report demonstrated a meaningful understanding of the concepts encapsulated in each learning outcome was evaluated. Reports were judged to have successfully achieved a learning outcome only if the report provided substantial evidence of a clear and complete understanding. For example, the description of a glass transition as "the temperature where the previously tangled chains begin to gain enough energy to move and slip around each other, leading to the material being able to be bent" was considered a clear demonstration of "understanding glass transitions" because it provided a clear statement of both what a glass transition is (temperature beyond which a material becomes less rigid) and why this transition occurs in some polymers. In contrast, cursory or superficial definitions lacking depth and/or explanation of why a phenomena occurs - for example "[a] glass transition is where a polymer changes from a harder and rigid material into a more flexible, rubbery material" - were not considered a clear demonstration of success in achieving a learning outcome. Note that this implies that reports containing completely correct definitional statements about polymer concepts could and were judged not to have successfully demonstrated various learning outcomes, because while the report may have demonstrated a memorization of definitions, it did not clearly demonstrate understanding of a concept. In addition, if a report did not discuss a particular aspect in meaningful detail - e.g., the "difference between polymerization and crosslinking" or "safety procedures for volatile organics," the report was deemed not a successful demonstration of the associated learning outcome.

\section{RESULTS}

\section{Hands-On Demo (MSE 101)}

Survey results for approximately two-thirds of demo participants (83 responses) were received following the Fall 2014 demo. Eighteen percent of respondents were materials engineering majors, while $82 \%$ of respondents were chemical engineering majors - a similar proportion to the ratio of materials versus chemical engineering freshman students who participated. Students were asked to indicate their overall satisfaction with the demo experience on a five-point scale, with 1 indicating a "very negative experience" and 5 indicating a "very positive experience." Responses averaged 3.9, indicating an overall positive student experience.

Students were asked: "How much did the polymers handson experience help you learn about (1) polymers, and (2) materials science and engineering as a discipline?" Response options were on a four-point scale, with "Not at all," "A little bit," "Some," and "A lot" equating to 1 through 4, respectively. Student responses averaged 2.9 and 2.8 for "polymers" and "MSE as a discipline," respectively, indicating that students felt they learned "some" about each topic.

When asked: "Would additional hands-on experiences enhance your MSE 101 or CME 101 course?" students overwhelmingly (85\%) responded "Yes." In addition, 31 open responses were provided to the prompt: "Please provide any comments or recommendations." Responses were overwhelmingly positive (>87\%), with recurring themes including:

- A desire for more lecture time to better explain polymers prior to the lab.

- A desire for more hands-on experiences in the introductory "101" courses.

- A desire for more time/discussion interaction than the 50 minute station-to-station process allowed.

Follow-up evaluation of a random sample of 20 one-page brief reports submitted by participating students highlighted both positive and negative aspects of the hands-on demo. First, the overall quality of the written reports was judged to be poor, with reports that were frequently poorly formatted, demonstrating poor English grammar and usage, and/or failing to venture beyond a basic description of the lab activities. Despite the students' self-assessments that they had learned "some" about both polymers and materials engineering as a discipline, very few students demonstrated meaningful understanding of the topics encompassed by the learning outcomes listed above.

Based on a holistic evaluation of survey results, student open-response comments, and the one-page brief reports, it is clear that the hands-on demo (MSE 101) version was very successful at motivating and engaging students, but the single 50-minute lecture in combination with the 50-minute hands-on demo were insufficient - on their own - to achieve the robust learning outcomes described above. While students were clearly "introduced" to polymers and polymer processing and pleased with the experience, follow-up coursework in later classes would be required to achieve any deep understanding of related concepts. Overall, these outcomes are precisely in line with the intent of the hands-on demo (MSE 101) version, and the department continues to offer the experience as an introduction, outreach, and engagement tool. 


\section{Laboratory Investigation (MSE 202)}

Twenty-three lab reports were submitted for the Fall 2015 MSE 202 Polymers lab, and a random subset of 15 were evaluated against the learning outcomes discussed above. Table 1 shows the findings. Note that the "\% Success" column describes how many of the reports out of the 15 evaluated successfully demonstrated achievement of the particular learning objective.

A majority (8+) of student reports demonstrated clear understanding of three of the eight criterion-understanding of the structure of polymer chains, polymer films, functional groups, and cross-links; understanding of tensile testing; and basic mixing and measuring procedures. Although not a majority, a notable number of student reports (4-6), exhibited understanding of the general mechanism of photo-catalyzed reactions, understanding of the glass transition temperature, and the role of polymer structure/crosslinking in controlling viscosity and film mechanical properties. Substantial numbers of additional reports gave correct definitions of concepts covered by the learning outcomes, but did not demonstrate clear and complete understanding (see discussion of evaluation criteria, above). In addition, the lab reports were un-prompted, and the writers of many otherwise excellent reports chose not to address all points covered by the learning outcomes identified for the overall polymers demos/lab. As noted above, if a report did not substantially address a point, it was deemed to not to have succeeded.

Overall, results for learning outcomes from the MSE 202 full lab version of the experience reinforce results for the MSE 101 demo version: the hands-on experience itself is not sufficient to give students a full and clear understanding of polymer science, but the experience provides an excellent introduction to the topic/field. In the context of the MSE 202 lab course, which is taken by sophomores while taking a textbook-based lecture overview course, this is ideal, as the experience serves as an engaging introduction to and preview of the full-semester course in polymer materials offered in the department. This 400-level course is required of all MSE majors in their junior year, and is taken as an elective by 10 $15 \mathrm{CME}$ juniors or seniors each year.

\section{Cost and Scalability}

Table 2 lists the chemicals and equipment used in the hands-on experience, including approximate costs and sources for equipment and supplies specific to the hands-on experience.

The setup used here consisted of two UV curing stations, 10 pairs

Vol. 52, No. 1, Winter 2018 of UV googles, a gross of $7.5 \mathrm{~cm}$ by $5 \mathrm{~cm}$ glass slides (each slide coated with RainX on one side), and two $3.5 \mathrm{oz}$. bottles of RainX, and is more than sufficient to handle 140+ students at $\sim 22$ students/hour for the demo version, and $\sim 15$ students divided into four lab groups continuously for the full lab version. Total initial setup costs, excluding recurring costs for polymer precursors, were $\sim \$ 350$ for the setup described here. For the demo version of the experience, the recurring costs for the polymer precursors (see Procedures, above, for sources) come to a total of approximately $\$ 0.92 /$ group, or between $\$ 0.19$ and $\$ 0.23 /$ student for groups of five or four students, respectively. Recurring costs for polymer precursors for the full lab version are approximately 10x as high due to the increased number of polymer batches made during exploration of different polymer recipes.

Lab scalability is limited by the number of fume hoods available. In delivering the demo version to $\sim 150$ students over just under 7 hours total, three fume hoods were used - one each for the Mixing, Casting, and UV Irradiation stations. Two USpicy UV sources were placed in the UV irradiation hood, allowing groups to overlap during UV curing, if necessary. This allowed six groups of four students each to circulate smoothly through the stations

\begin{tabular}{|l|c|}
\hline \multicolumn{2}{|c|}{ TABLE 1 } \\
\hline \multicolumn{1}{|c|}{ Outcome } & $\%$ Success \\
\hline Basic mixing and measuring procedures & 67 \\
\hline $\begin{array}{l}\text { Understanding of the structure of polymer } \\
\text { chains, polymer films, functional groups, and } \\
\text { cross links }\end{array}$ & 53 \\
\hline Understanding of tensile testing & 53 \\
\hline Understanding of glass transition & 40 \\
\hline $\begin{array}{l}\text { Role of polymer structure/crosslinking in con- } \\
\text { trolling viscosity and film mechanical properties }\end{array}$ & 27 \\
\hline $\begin{array}{l}\text { Understanding of the general mechanism of } \\
\text { photo catalyzed reactions }\end{array}$ & 27 \\
\hline Effective presentation of technical information & 20 \\
\hline $\begin{array}{l}\text { Difference between polymerization and cross- } \\
\text { linking }\end{array}$ & 7 \\
\hline Safety procedures for volatile organics & 7 \\
\hline
\end{tabular}

TABLE 2

Equipment and supplies required for the polymers hands-on experience and lab

\begin{tabular}{|l|l|l|}
\hline \multicolumn{2}{|c|}{ Polymers experience-specific equipment } & General lab equipment \\
\hline USpicy 36W Nail Dryer & $\$ 40 /$ each (including bulbs), Amazon.com & Fume hoods \\
\hline UV Goggles & $\$ 113 /$ ten pairs, VWR & Heat gun \\
\hline 4"x6" glass panels & $\$ 0.69 /$ panel, Frames.com & Sink w/ soap and water \\
\hline $7.5 \mathrm{~cm} \times 5 \mathrm{~cm}$ glass slides & $\$ 58 / 144$ slides, Fisher & \\
\hline RainX, 3.5 oz. & $\$ 5$, Amazon.com & \\
\hline 1'x 2' Teflon sheet & $\$ 52 /$ sheet, McMaster-Carr & \\
\hline Binder clips & $\$ 10 / 100$, Amazon.com & \\
\hline
\end{tabular}


per hour, or, on average, 22/students per hour. Additional fume hoods would enable "doubling up" the number of stations, or, alternatively, a single fume hood could be used to house all three of the Mixing, Casting, and UV Irradiation "stations." Under a single fume hood model, it is estimated that two groups at a time could be distributed over the three "stations" requiring the fume hood, for a reduced rate of $\sim 14$ students per hour (two-thirds of that achieved with the separate hood model used here). Note that because overall it takes students approximately 50 minutes to complete the experience, the throughput rates cited here only apply once students begin finishing the experience. Ultimately this implies that the minimum useful time window for moving large numbers of students (10+) through the demo experience is close to two hours.

Similarly, scalability of the full lab version is primarily limited by the number of fume hoods that can be used at one time. One fume hood and one or two UV systems per lab group are recommended for the full lab version.

\section{CONCLUSION}

The experiences described here succeeded as engaging hands-on introduction to polymers and polymer processing for both chemical and materials engineering freshmen, as well as for sophomore materials engineers in their first engineering lab course. The experience demonstrated the interconnectedness of processing, structure, and properties, and brought chemical and materials engineering students together at an intersection of their respective disciplines. The hands-on demo proved that students could fabricate a rigid polymer from liquid precursors using an inexpensive acrylic nail lamp at high student-throughput rates. Students could be exposed to the concepts of crosslinking and glass transition in a less formal setting than the traditional lecture, allowing students to actually experience the concepts instead of solely hearing them described.

Student satisfaction with the demo version of the experience was very high, and offered substantial evidence that the experience motivated student interest in more polymers and polymer processing classroom content. Neither the demo version nor the full lab experience provided students with a clear and complete understanding of the broad set of polymers-related learning objectives evaluated as part of this study. Despite this, both the demo and full lab version of the experience are excellent complements to traditional lectures.

\section{APPENDIX}

\section{Initial Evaluation of Activation Mechanism}

During Fall 2015 the specific method of activating the DMPA catalyst was evaluated. In 2013 and 2014, a single continuous-wave, broad-spectrum UVA flood lamp (max flux at $365 \mathrm{~nm}$ ) had been used to photo-activate the DMPA catalyst to induce cross-linking and polymer film solidification. As the number of participants expanded to $\sim 140$ freshman students, it became necessary to acquire additional activation stations. An alternative thermal activation process using a commercial microwave oven has been described by Lee, et al. ${ }^{[28]}$ for crosslinking essentially the same co-polymer blend. The method described used a silicon wafer to convert microwaves into heat, which then induced DMPA-catalyzed cross-linking, and subsequent polymer film formation, thermally.

To test this activation approach, a commercial 1000W Hamilton Beach microwave was used, along with silicon wafers [2" diameter, P (100), 1x10-10 $\Omega / \mathrm{cm} \mathrm{SSP,} 380 \mu \mathrm{m}$ thickness, purchased from University Wafer], following the procedure described by Lee, et al. ${ }^{[28]}$ Despite extensive exploration of cook times and power levels, we were unable to form uniform, coherent, solid polymer films. Operating the microwave with the silicon wafer in place repeatedly and reproducibly generated sparks in the oven, but did not repeatedly or reproducibly result in controlled temperature increases of the wafer, or a Pyrex slide placed atop the wafer, or a polymer blend atop the wafer. While it was possible to produce solid polymer "chunks" using this procedure, it was not possible to produce films of uniform thickness or stiffness, and the solid material also contained large number of bubbles, holes, and other imperfections. In some cases thermal stresses induced during microwave cooking were so severe as to spontaneously fracture Pyrex slides, the silicon wafer, or both. In a few cases, fracture of a Pyrex slide with polymer precursor atop it allowed the precursor to directly contact the wafer, resulting in combustion and flames in the oven.

These findings motivated a comprehensive study of silicon wafer behavior when exposed to microwaves. First the temperature in the empty microwave was measured before it was turned on, directly after it had been on for $15 \mathrm{~s}$, and then every minute until the interior of the oven returned to room temperature. It was found that even though each trial began at room temperature, the residual temperatures after 15 seconds of microwave operation at full power were different for each trial. It was also observed that it took several minutes for the interior to return to room temperature. Next, the temperature of the silicon wafer alone before and after microwaving was studied. Results were highly variable, with sparking occurring after apparently random microwave operation times ranging from 12 seconds to more than 1 minute. Wafer temperatures after microwave operation were random in their ranges and not reproducible. Finally the effects of heating a Pyrex slide atop a silicon wafer were considered. No reproducible results could be obtained, as sparking continued after apparently random intervals, and thermal stresses often resulted in fracture of the slides.

In a continuing attempt to increase the availability of cross-linking activation stations, a commercial UV system (USpicy 36W Nail Dryer/UV Lamp system, purchased from 
Amazon, \$40, bulbs included, bulbs indicate "365 nm" light) for curing nail polish was evaluated in comparison to a broad spectrum UVA flood lamp (365 nm max brightness). The results were immediately positive. Exposure to UV irradiation in the USpicy for 12-13 minutes (only 2-3 minutes more than the exposure required in the flood lamp system) resulted in consistent, uniform, solid polymer films. While the USpicy does not have a door (and therefore, users can be exposed to UV light), the commercial availability of the product, and the lack of printed warnings on the device or included manuals and instructions, suggest that the system is completely safe for student use. Despite this, during utilization the USpicy systems were turned to face the back of the hood, and students at the UV irradiation station were required to where UV-rated lab goggles. The USpicy systems utilize four $9 \mathrm{~W}$ U-shaped UVA bulbs ( $365 \mathrm{~nm})$, and replacement bulbs are widely available (e.g., on Amazon.com) for $\sim \$ 2.50 /$ bulb.

\section{Lab and Personal Protection Equipment}

Students participating in both the MSE 101 demo and MSE 202 full lab versions of the experience wore lab coats, standard safety googles, and nitrile (VWR) gloves. Students were required to wear closed-toe shoes, and have hair and all loose jewelry secured. Polymer mixing, mold filling, and film curing were conducted in fully ventilated fume hoods, and only stoppered flasks were allowed to be moved between stations.

\section{REFERENCES}

1. Moller-Wong, C., and A. Eide, "An Engineering Student Retention Study,” J. Eng. Educ., 86(1), 7 (1997)

2. Forsman, J., M. van den Bogaard, C. Linder, and D. Fraser, "Considering student retention as a complex system: a possible way forward for enhancing student retention," European J. Eng. Educ., 40(3), 235 (Oct. 2014)

3. Ohland, M.W., S.D. Sheppard, G. Lichtenstein, O. Eris, D. Chachra, and R.A. Layton, "Persistence, Engagement, and Migration in Engineering Programs," J. Eng. Educ., 97(3), 259 (2008)

4. Desai, N., and G. Stefanek, "A literature review of the different approaches that have been implemented to increase retention in engineering programs across the United States," Proceedings of the 2017 ASEE Zone II Conference, San Juan, Puerto Rico, 2017

5. Litzler, E., and J. Young, "Understanding the Risk of Attrition in Undergraduate Engineering: Results from the Project to Assess Climate in Engineering," J. Eng. Educ., 101(2), 319 (Jan. 2013)

6. Hewitt, N.M., and E. Seymour, "A Long, Discouraging Climb," ASEE Prism, 1(6), 24 (1992)

7. Fiske, S.T., J.G. Cromley, T. Perez, and A. Kaplan, "Undergraduate STEM Achievement and Retention," Policy Insights from the Behavioral and Brain Sciences, 3(1), 4 (Dec. 2015)

8. Atwood, S.A., and J.E. Pretz, "Creativity as a Factor in Persistence and Academic Achievement of Engineering Undergraduates," J. Eng. Educ., 105(4), 540 (Sept. 2016)
9. Carlson, B., P. Schoch, M. Kalsher, and B. Racicot, "Evaluating a motivational freshman course," Proceedings of the Frontiers in Education 1995 25th Annual Conference. Engineering Education for the 21st Century, Atlanta, GA, 1, 2a6.14 (1995)

10. Carlson, B., P. Schoch, M. Kalsher, and B. Racicot, “A Motivational First-year Electronics Lab Course,” J. Eng. Educ., 86(4), 357 (1997)

11. Higley, K.A., and C.M. Marianno, "Making Engineering Education Fun,” J.Eng. Educ., 90(1), 105 (Jan. 2013)

12. Felder, R.M., D.R. Woods, J.E. Stice, and A. Rugarcia, "The Future of Engineering Education: II. Teaching Methods That Work," Chemical Engineering Education, 34(1), 26 (2000)

13. Branch, S.E., A. Woodcock, and W. G. Graziano, "Person Orientation and Encouragement: Predicting Interest in Engineering Research," $J$. Eng. Educ., 104(2), 119 (Apr. 2015)

14. Hoit, M., and M. Ohland, "The Impact of a Discipline-Based Introduction to Engineering Course on Improving Retention," J. Eng. Educ., 87(1), 79 (1998)

15. Froyd, J.E., and M.W. Ohland, "Integrated Engineering Curricula," $J$. Eng. Educ., 94(1), 147 (2005)

16. de Jong, T., M.C. Linn, and Z.C. Zacharia, "Physical and Virtual Laboratories in Science and Engineering Education," Science, 340(6130), 305 (Apr. 2013)

17. Aglan, H.A., and S.F. Ali, "Hands-On Experiences: An Integral Part of Engineering Curriculum Reform," J. Eng. Educ., 85(4), 27 (1996)

18. Taajamaa, V., T. Westerlund, Xing Guo, M. Hupli, S. Salantera, and T. Salakoski, "Interdisciplinary engineering education - Practice based case," Fourth Interdisciplinary Engineering Design Education Conference, Santa Clara, CA, pp. 31-37 (2014)

19. Kresta, S.M., "Hands-on Demonstrations: An Alternative to Full Scale Lab Experiments,” J. Eng. Educ., 87(1), 7 (1998)

20. Jensen, D., J. Wood, and K. Wood, "Hands-on Activities, Interactive Multimedia and Improved Team Dynamics for Enhancing Mechanical Engineering Curricula," Int. J. Engng Ed., 19(6), 874 (2003)

21. Major, D.A.,V.N. Streets, D.P. Myers, and R.E. Green, "A Passionate Approach to Recruiting and Retaining Engineering Research Participants," J. Eng. Educ., 105(3), 401 (May 2016)

22. Lohmann, J.R., H.A. Rollins, and J.J. Hoey, "Defining, developing and assessing global competence in engineers," European J. Engineering Education, 31(1), 119 (Mar. 2006)

23. Black, K.M., “An Industry View of Engineering Education,” J. Eng. Educ., 83(1), 26 (1994)

24. Sheppard, K., P. Dominick, and Z. Aronson, "Preparing Engineering Students for the New Business Paradigm of Intern," Int. J. Engng Ed., 20(3), 475 (2004)

25. Telenko, C., K. Wood, K. Otto, M. Rajesh Elara, S. Foong, K. Leong Pey, U.-X. Tan, B. Camburn, D. Moreno, and D. Frey, "Designettes: An Approach to Multidisciplinary Engineering Design Education," $J$ Mech. Des., 138(2), 022001, (Feb. 2016)

26. Borrego, M., and L.K. Newswander, "Characteristics of Successful Cross-disciplinary Engineering Education Collaborations," J. Eng. Educ., 97(2), 123 (2008)

27. Hager, M.D., S. Bode, C. Weber, and U.S. Schubert, "Shape memory polymers: Past, present and future developments," Progress in Polymer Science, 49, 3 (2015)

28. Lee, S.H., W.G. Lee, B.G. Chung, J.H. Park, and A. Khademhosseini, "Rapid Formation of Acrylated Microstructures by Microwave-Induced Thermal Crosslinking," Macromolecular Rapid Communications, 30(16), 1382 (2009) $\square$ 